\title{
Sleep disordered breathing in patients with heart failure
}

\author{
Magdalena Krawczyk ${ }^{1}$, Irena Flinta ${ }^{1}$, Magdalena Garncarek ${ }^{1}$, Krystian Josiak ${ }^{1,2}$, \\ Ewa Anita Jankowska, ${ }^{1,2}$, Waldemar Banasiak ${ }^{1}$, Robin Germany ${ }^{3,4}$, \\ Shahrokh Javaheri ${ }^{5}$, Piotr Ponikowski ${ }^{1,2}$ \\ ${ }^{1}$ Heart Diseases Center, $4^{\text {th }}$ Military Hospital, Wroclaw, Poland \\ ${ }^{2}$ Department of Heart Diseases, Medical University, Wroclaw, Poland \\ ${ }^{3}$ The University of Oklahoma, Oklahoma City, OK, USA \\ ${ }^{4}$ Respicardia, Inc., Minnetonka, MN, USA \\ ${ }^{5}$ University of Cincinnati College of Medicine, and Sleepcare Diagnostics, Cincinnati, OH, USA
}

\begin{abstract}
Sleep disordered breathing (SDB) is a common co-morbidity in patients with heart failure (HF). Both its forms - central and obstructive sleep apnea - are highly prevalent in this population. SDB fragments sleep, impairs life quality, worsens exercise tolerance, worsens $H F$ and is an independent predictor of poor prognosis. Still, SDB remains largely undiagnosed. Therefore, early detection of SDB seems to be of primary importance, especially in the presence of new diagnostic and therapeutic methods. Treatment with continuous positive airway pressure (CPAP) increases left ventricular ejection fraction and stroke volume in virtually all HF patients with obstructive and in $50 \%$ with central sleep apnea. For those in whom central sleep apnea is not suppressed by CPAP, a trial of adaptive servoventilation is recommended. Although no randomized, controlled trials have shown improvement in mortality, several observational studies have shown that effective treatment of both forms of sleep apnea with various positive airway pressure devices improves survival of HF patients. Currently, 2 large trials with newer masked based therapies with adaptive servoventilation are in progress. This article is a brief overview of present knowledge, the pathophysiology, diagnostic approach and therapy of SDB. (Cardiol J 2013; 20, 4: 345-355)
\end{abstract}

Key words: central sleep apnea, Cheyne Stokes respiration, heart failure

\section{Introduction}

Sleep disordered breathing (SDB) is one of the most common and yet largely under-diagnosed co-morbidity in patients with heart failure (HF) $[1,2]$. It leads to further deterioration of cardiac function and worsens prognosis [3-6]. SDB may be a potential therapeutic target as its effective treatment improves quality of life, exercise tolerance and, possibly, long term prognosis [5-10]. However, the importance of SDB remains unrecognized and the disorder itself is diagnosed rarely and remains untreated [5]. The availability of simple screening methods and severe clinical consequences of SDB justify routine and widespread screening and appropriate treatment.

Address for correspondence: Magdalena Krawczyk, MD, Heart Diseases Center, $4^{\text {th }}$ Military Hospital, ul. Weigla 5, 50-980 Wroclaw, Poland, e-mail: krawmag@gmail.com

Received: 03.12.2012 Accepted: 28.03.2013 


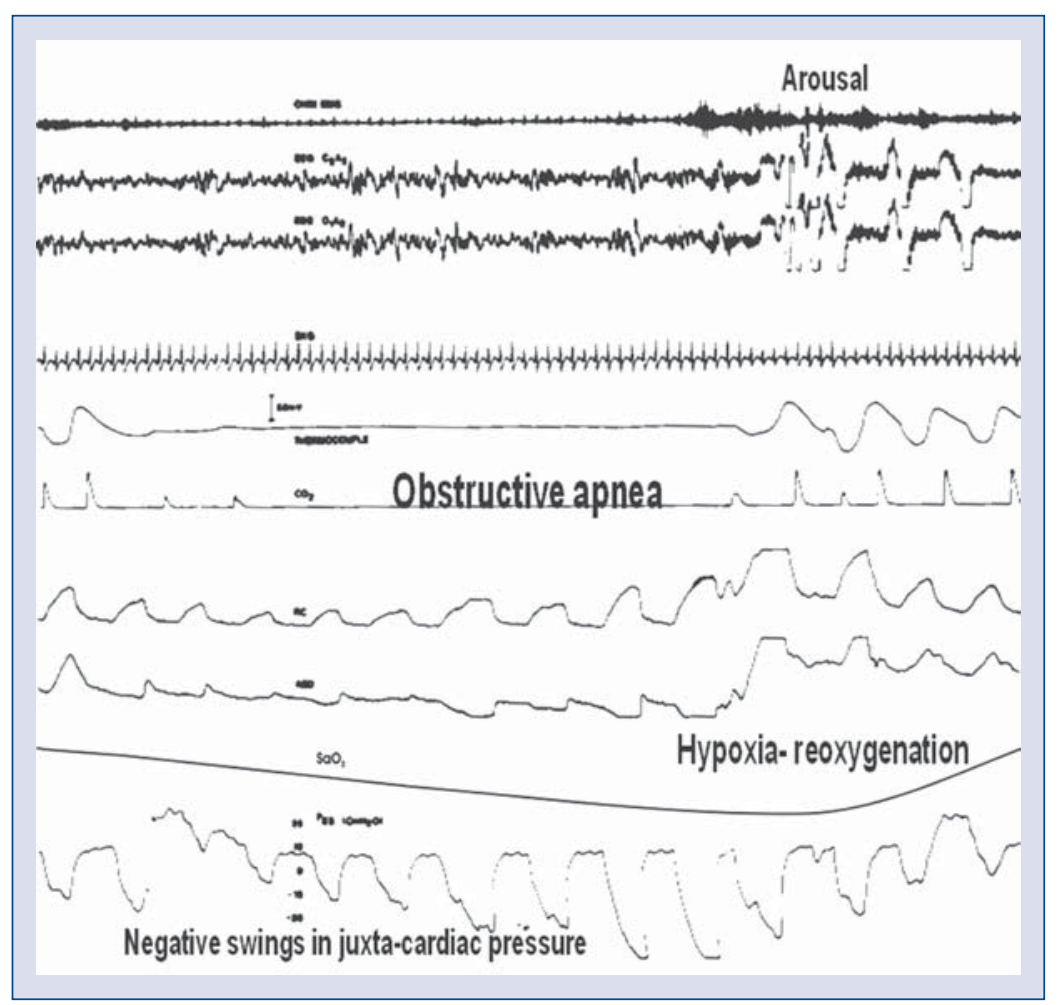

Figure 1. Acute consequences of obstructice sleep apnea (OSA). An example of OSA in a patient with heart failure. Note that the apnea is terminated with an arousal seen on brain waves. Also note progressively increasing negative intrathoracic pressure swings measured by an esophageal balloon. The thoracoabdominal excursions are out of phase during the apnea, but in phase during recovery. Also note desaturation with apnea and reoxygenation with resumption of breathing. Modified from: Javaheri S. Cardiovascular disorders. In: Kryger MH ed. Atlas of clinical sleep medicine. WB Saunders, Philadelphia 2010.

\section{Diagnosis and definitions}

Overnight polysomnography (PSG) is the gold standard for diagnosing SDB. These studies are completed in a sleep laboratory with monitoring. During a sleep study, breathing disorders can be detected and differentiated into central (CSA) and obstructive sleep apnea (OSA), based on presence of respiratory effort. Multiple parameters are monitored including oro-nasal airflow, thoracoabdominal movements, arterial blood oxyhemoglobin saturation, electrocardiogram (ECG), at least 2 channel electroencephalogram (EEG), chin and anterior tibialis electromyogram (EMG) and electrooculogram (EOG). With this montage total recording time, total wake time, total sleep time, sleep stages, apnea/hypopnea index (AHI) and arousal index are accurately determined.

Apnea is defined as a cessation of respiratory airflow for $10 \mathrm{~s}$ or more. If respiratory effort, manifested by abdominal and/or chest wall movement, is present, OSA is diagnosed (Fig. 1). CSA is diagnosed when no respiratory muscles activity is detec- ted (Fig. 2). Hypopnea is defined as the reduction in airflow by at least $50 \%$ of the preceding baseline associated with the drop in arterial blood oxygen saturation of at least $3 \%$. An alternative definition of hypopnea is reduction of airflow by at least $30 \%$ and associated with $4 \%$ or more desaturation. The number of apnea and hypopnea episodes during $1 \mathrm{~h}$ of sleep, known as AHI, is a valuable tool in recognition of sleep disorders and determination of their severity. Normally AHI should not exceed $5 / \mathrm{h}$. An AHI of 5 to $<15 / \mathrm{h}$ of sleep is considered mild, $\geq 15$ to $<30 / \mathrm{h}$ moderate and $\geq 30 / \mathrm{h}$ severe SDB [11]. Disordered breathing events are also associated with arousals. The number of arousals per hour of sleep is calculated as the arousal index.

Due to low accessibility and high cost of PSG new diagnostic methods have been developed. Self-assessment questionnaires, such as the Epworth Sleepiness Scale or Berlin Questionnaire, estimating the probability of SDB presence based on subjective symptoms are in common use. These questionnaires have been successfully used in general population, but as noted below they are 


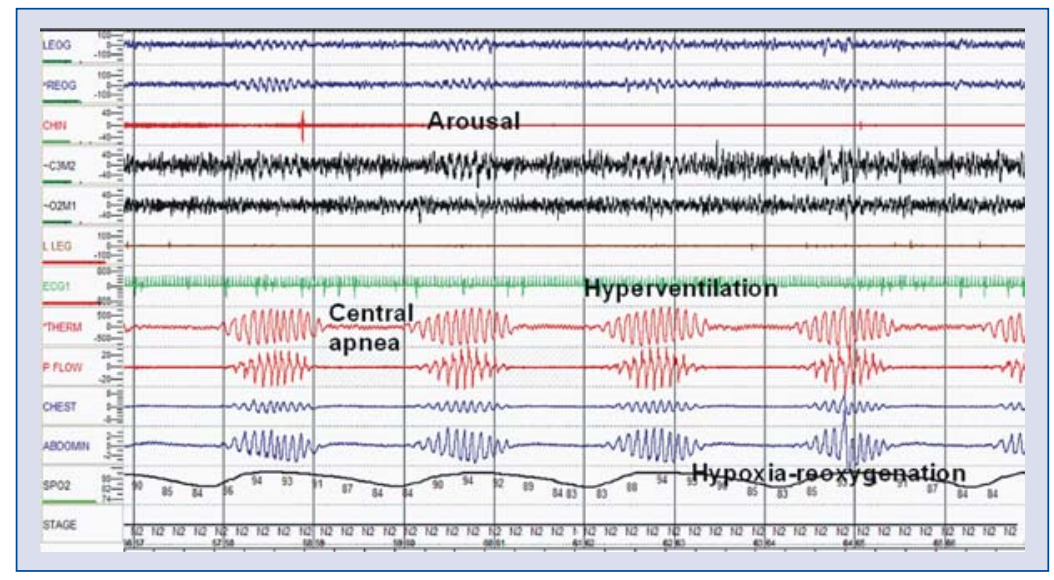

Figure 2. Hunter-Cheyne-Stokes breathing in heart failure. An example of periodic breathing in a patient with heart failure in N2 (nonrapid-eye-movement sleep). Note crescendo decrescendo changes in tidal breathing which sandwich central apneas. Also, note that arousals occur at the peak of hyperventilation. This contrasts with the temporal association of arousals occurring at the termination of obstructive apneas (see Figure 2). Modified from: Javaheri S. Cardiovascular disorders. In: Kryger MH ed. Atlas of clinical sleep medicine. WB Saunders, Philadelphia 2010.

virtually useless for suspicion of sleep apnea in HF patients.

There are numerous clinical findings that may suggest OSA in general population, such as snoring, interrupted sleep, increased daytime sleepiness, morning headaches, nocturia, chronic fatigue, arterial hypertension, and arrhythmias. A number of these symptoms such as interrupted sleep, nocturia, fatigue and arrhythmias are nonspecific, being present in HF itself. Furthermore, in patients with HF, the prevalence of excessive daytime sleepiness is similar between those with or without sleep apnea $[12,13]$. For all these aforementioned reasons, sleep apnea remains under diagnosed in HF [5]. Snoring, however, remains a predictor of OSA in patients with $\mathrm{HF}$ as it is, in general population. Similarly, hand in hand with snoring, obesity remains a major risk factor for OSA in HF, and its prevalence is on the rise in this population.

Diagnosis of CSA in patients with HF is most problematic as HF patients with CSA are generally thin and do not snore much [12]. Major predictors of CSA are atrial fibrillation, very low left ventricular ejection fraction (LVEF less than 20\%), presence of nocturnal ventricular arrhythmias and a low steady state arterial $\mathrm{PCO}_{2}[13]$.

Recently, new methods which seem to be useful in identifying patients with SDB have appeared. One of them utilizes heart rate variability (HRV) assessment. A typical pattern of HRV with very low frequency rhythms has been found in patients with $\mathrm{HF}$ and SDB, especially with CSA (for review see reference [14]). This can be measured at home with a Holter monitor, but is not diagnostic for the type or severity of SDB. Another method that can be applied in patients with implanted pacemakers, relies on the assessment of minute ventilation by evaluation of changes in transthoracic impendence between the pacemaker case and the electrode tip. It enables detection of decreased ventilation episodes. However, it cannot differentiate between obstructive and central episodes. Importantly, this method showed high sensitivity in identifying severe SDB when compared to traditional PSG, suggesting its potential application as a screening tool in patients with implantable cardiac devices [15].

Home sleep-studies with cardiorespiratory polygraphy have been utilized for diagnosis of sleep apnea both in general population and in patients with HF. A major the difference with in laboratory PSG is lack of EEG. There are several portable devices available: Embletta, ApneaLink, Stardust, Venla, to name a few. Typically, they consist of nasal pressure detector, belts detecting thoraco-abdominal movement and finger pulse oximeter. They have some potential advantages over PSG, such as lower cost, better sleep quality in home environment than in sleep laboratory, portability and broad accessibility. However, home sleep-studies cannot replace full PSG as for lack of EEG, total sleep time cannot be determined. As a result, the true $\mathrm{AHI}(\mathrm{AHI} / \mathrm{h}$ of sleep) is underestimated, as the denominator used for the calculation is the total recording time, and not the hours of sleep. This is an important consideration, since patients with $\mathrm{HF}$ 
Table 1. Prevalence of sleep apnea $(A H I \geq 15 / h)$, central (CSA) and obstructive (OSA) sleep apnea in recent prospective studies in patients with heart failure with reduced ejection fraction; NA — not available. Modified from: [2].

\begin{tabular}{lccccc}
\hline Country [year] Author & N & AHI $\geq \mathbf{1 5 / h}$ & CSA & OSA & $\beta$-blockers \\
\hline *USA [2006] Javaheri & 100 & $49 \%$ & $37 \%$ & $12 \%$ & $10 \%$ \\
USA [2008] MacDonald & 108 & $61 \%$ & $31 \%$ & $30 \%$ & $82 \%$ \\
*Canada [2007] Wang & 287 & $47 \%$ & $21 \%$ & $26 \%$ & $80 \%$ \\
*UK [2007] Vazir & 55 & $53 \%$ & $38 \%$ & $15 \%$ & $78 \%$ \\
Germany [2007] Oldenburg & 700 & $52 \%$ & $33 \%$ & $19 \%$ & $85 \%$ \\
${ }^{*}$ Germany [2009] Hagenah & 50 & $64 \%$ & $44 \%$ & $20 \%$ & $100 \%$ \\
${ }^{*}$ Germany [2010] Jilek & 273 & $64 \%$ & $50 \%$ & $14 \%$ & $88 \%$ \\
*Portugal [2010] Ferreira & 103 & $46 \%$ & NA & NA & $90 \%$ \\
Total & 1676 & $54 \%$ & $34 \%$ & $20 \%$ & $81 \%$ \\
\hline
\end{tabular}

${ }^{*}$ Asterisk indicates studies using polysomnography.

have curtailed sleep time even after spending 2 consecutive nights in sleep laboratory for habituation $[12,13]$. Therefore, a number of HF patients with SDB may go unrecognized as a negative polygraphy does not rule out significant sleep apnea.

\section{Epidemiology of SDB in HF}

The prevalence of SDB has been extensively studied both in patients with reduced as well as preserved LVEF. In the population of patients with $\mathrm{HF}$ and reduced left ventricular ejection fraction, SDB occurs frequently. Multiple studies (Table 1) show a high prevalence of sleep apnea both central and obstructive in this population [13, 16-20]. One important difference is the variability in the distribution of central vs. obstructive disordered breathing events. This difficulty stems from accurately differentiating obstructive and central sleep hypopneas from each other. Combining the results of multiple studies from various countries, the overall prevalence of sleep apnea in patients with $\mathrm{HF}$ with a threshold of an AHI of 15 or more per hour is $52 \%$. Central apnea accounts for $32 \%$ and obstructive apnea for $20 \%$ (Table 1). It is important to emphasize that the subjects enrolled in most recent studies were consecutive patients with stable HF on contemporary therapy. Therefore, the results of these studies are consistent with those of early studies in which only $10 \%$ of the patients were on a beta-blocker (Table 1). We therefore conclude that introduction of beta-blockers for treatment of $\mathrm{HF}$ has had no major impact on the prevalence of sleep apnea in this population.

There is growing evidence that sleep apnea is also highly prevalent in patients with $\mathrm{HF}$ and preserved ejection fraction. Bitter et al. [21] used echocardiography and right and left heart catheterization to characterize sleep apnea in 244 consecutive patients with $\mathrm{HF}$ and preserved ejection fraction. In this study, 33\% had coronary artery disease, $44 \%$ had systemic hypertension and $23 \%$ had hypertrophic/restrictive cardiac disease. Using polygraphy without EEG, $48 \%$ of the patients had an $\mathrm{AHI} \geq 15 \mathrm{~h}$ of recording time. As noted earlier, polygraphy may underestimate the severity of sleep apnea, therefore the data from patients with $\mathrm{HF}$ and preserved ejection fraction are virtually similar to those in patients with $\mathrm{HF}$ and reduced ejection fraction. Twenty-five percent of the 244 patients had OSA and 23\% had CSA.

\section{Pathomechanisms of SDB}

\section{Central sleep apnea}

Central sleep apnea occurs in the background of a specific respiratory pattern known as Hunter-Cheyne-Stokes breathing (HCSB). It had first been described by John Hunter [22], 37 years before it was described by Cheyne in 1818 [23] (see references [1] and [2] for more details). HCSB is characterized by recurrent episodes of apnea/hypopnea in the context of long crescendo-decrescendo breathing cycles. The length of the cycle reflects the prolonged circulation time, the pathologic feature of HF. HCSB occurs in patients with HF both during the day and at night, but CSA occurs only during sleep or when the patient is dozing. Pathological mechanisms underlying CSA have been widely investigated within last decade and explained in detail in a recent monograph [24]. The important factors involved in pathophysiology of CSA are: diminished $\mathrm{PCO}_{2}$ reserve (difference between eupneic $\mathrm{PCO}_{2}$ and apnea threshold) [24, 
25], and augmented central and peripheral hypoxic and hypercapnic chemosensitivity [26, 27]. These will be discussed in details below (Fig. 2).

Hyperventilation is a consequence of chronic pulmonary congestion and interstitial pulmonary edema. In response to pulmonary congestion vagus nerve becomes activated (by non-myelinated type $\mathrm{C}$ nerve fibers in parenchyma of the lungs, in alveolar bronchioles, alveolar capillaries and distention-sensitive receptors), that leads to central activation of breathing. Meanwhile, pulmonary congestion also decreases the $\mathrm{PCO}_{2}$ reserve and predisposes to central apnea [24], this by mechanisms not fully explained. Therefore, during sleep, even a slight increase in ventilation caused by arousal or changes in position may lead to a drop in $\mathrm{PCO}_{2}$ below the apnea threshold and results in occurrence of apnea episode. Cessation of breathing leads to accumulation of $\mathrm{CO}_{2}$ which is the chemical stimulus for resumption of ventilation. Enhanced chemoreceptor sensitivity propagates excessive ventilation and subsequent drop in $\mathrm{PCO}_{2}$ below apnea threshold and the next apnea $[1,2,24,26]$. The duration of the cycle of periodic breathing is proportional to circulation time from lung to chemoreceptors.

In $\mathrm{HF}$ patients with $\mathrm{CSA}, \mathrm{PCO}_{2}$ does not increase at sleep onset which narrows $\mathrm{PCO}_{2}$ reserve increasing the likelihood of developing CSA. It has been suggested $[2,24]$ that lack of increase in $\mathrm{PCO}_{2}$ results from inability to decrease ventilation that normally occurs at sleep onset. Patients with HF, particularly those with severely impaired diastolic function, may develop an increase in pulmonary capillary pressure due to increased venous return, which in turn reflexively increases respiratory rate and ventilation at sleep onset. Therefore, nocturnal rostral shift of fluid into the lung causing pulmonary congestion enhances CSA [28].

Regarding hypocapnia 2 points are emphasized. First, in HF awake steady state arterial hypocapnia is highly predictive of CSA [29]. However, in a group of patients with liver cirrhosis and preserved ejection fraction (mean $\mathrm{EF} 60 \%$ ), despite hypocapnia (mean arterial $\mathrm{PCO}_{2}=32 \mathrm{~mm} \mathrm{Hg}$ ), central apneas were rarely observed. On the other hand hypocapnia in HF subjects with impaired ejection fraction (mean EF 23\%) was associated with increased number of AHI episodes and central apneas [30]. Thus, it may not be hypocapnia itself, but diminished difference between eupneic $\mathrm{PCO}_{2}$ and apnea threshold, known as $\mathrm{PCO}_{2}$ reserve that predisposes to CSA, as discussed earlier [24, 25].
Another factor which may increase the likelihood of developing central apnea is cerebrovascular response to $\mathrm{PCO}_{2}$ which may result in reduced $\mathrm{PCO}_{2}$ reserve. To this end Xie et al. [31] used indomethacin to reduce cerebral blood flow response to $\mathrm{PCO}_{2}$ and using a mechanical ventilator in pressure support mode gradually reduced $\mathrm{PCO}_{2}$ until apnea occurred. They observed that in conditions of decreased cerebrovascular reactivity, $\mathrm{PCO}_{2}$ reserve needed to produce an apnea dropped from 6.3 to $4.4 \mathrm{~mm} \mathrm{Hg}$ [31]. This is an important observation as cerebral blood vessels reactivity to $\mathrm{CO}_{2}$ is diminished in patients with $\mathrm{HF}$ [32], which may lead to a significant decrease in $\mathrm{PCO}_{2}$ reserve and consequently promote breathing instability.

\section{Obstructive sleep apnea}

The pathomechanisms of OSA are complex and their detailed description remains beyond the goal of this article, however they have been discussed in detail elsewhere [33]. The main underlying mechanism of OSA is partial or complete occlusion of upper airway, mainly due to genioglossus muscle relaxation in the face of altered mechanical properties of the upper airway. With this background, negative pressure within airways generated by diaphragm contraction during inspiration results in pharyngeal collapse and obstruction.

Factors that impair upper airway patency relate to upper airway anatomical abnormalities such as micrognathia, retrognathia, hypertrophy of palatine tonsils, macroglossia and increased adipose tissue deposition. In patients with HF, rostral fluid shift in supine position causing venous congestion and upper airway edema may further predispose to upper airway collapse [28].

We must emphasize however, that most frequently both types of apnea occur together during one night. This was recognized early on for which reason we used arbitrary criteria to define whether the breathing disorder is predominantly central or obstructive in nature [12, 13, 34]. Presence of a hybrid disordered breathing and the predominant form of sleep apnea depends on a number of factors such as use of alcohol, medications, changes in position and body weight and fluid shifts.

\section{Clinical consequences}

Normally, about $80 \%$ of the sleep period is non-rapid eye movement sleep (NREM), when increased parasympathetic activity and decreased sympathetic activity is observed. This is associated with a drop in heart rate, cardiac output and 


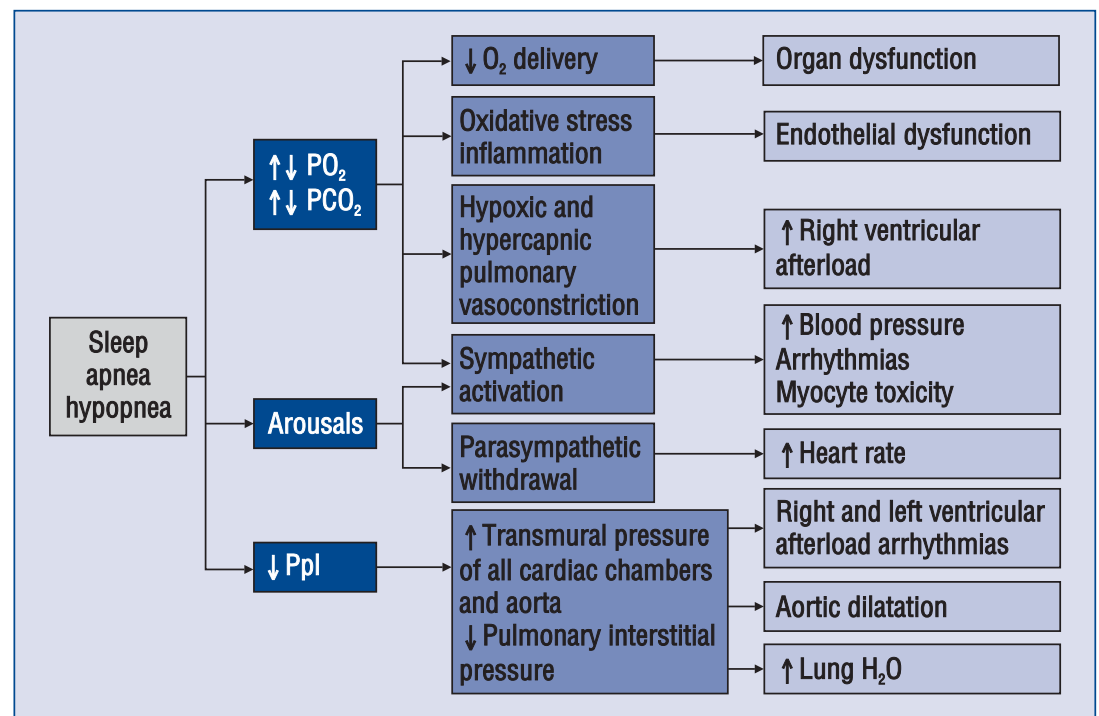

Figure 3. Biological pathways mediating cardiovascular complications of sleep apnea; Ppl — pleural pressure. Modified from [2].

blood pressure, therefore, there is cardiovascular quiescence. However, in the face of sleep apnea, NREM sleep is fragmented with arousals, and along with other consequences of sleep apnea, a hyperadrenergic state is created $[1,2]$.

We must emphasize that the biological consequences of sleep apnea which adversely affect cardiovascular function are qualitatively similar in both forms of apnea although such adverse effects are more severe in OSA than CSA (Fig. 1).

\section{Consequences of sleep apnea in HF}

The SDB events are characterized by cycles of apnea/hypopnea followed by recovery. The 3 main consequences of SDB are hypoxia/hypercapnia, negative swing in intrathoracic pressure and arousals (Fig. 1-3). During recovery following apneas or hypopneas, ventilation increases and reoxygenation/hypocapnia ensue. These consequences of sleep apnea are qualitatively similar when the 2 forms of apnea are compared; however, they are more pronounced in OSA than CSA (Fig. 3).

A major adverse consequence of apneas/hypopneas is elevated sympathetic nervous system activation induced by repetitive arousals and hypoxia/ hypercapnia (Fig. 3) through enhanced peripheral and central chemoreceptors $[1,24,26,27,35]$. Increased sympathetic activity leads to vasoconstriction and subsequently to a surge in blood pressure, heart rate and left ventricular afterload - changes that are particularly unfavorable in patients with HF. As a consequence of elevated sympathetic activation nocturnal arrhythmias may also occur [11, 13, 29, 34]. Most recently [36], it has been shown that in HF patients with sleep apnea, current smoking further increases the odds ratio for nocturnal arrhythmias considerably; nicotine, via activation of the peripheral chemoreceptors, a shared pathway with hypoxia, increases sympathetic activity and this hand in hand, with other consequences of sleep apnea contribute to excess nocturnal arrhythmias in HF [39], creating the substrate for sudden cardiac death, a major mode of mortality in these patients. Cessation of smoking should have a major impact on prevention of sudden cardiac death in HF patients, particularly those with sleep apnea as it does in patients with coronary artery disease.

Hypoxia and hypercapnia lead to vasoconstriction not only in systemic but also in pulmonary circulation resulting in augmented right ventricle afterload (Fig. 3). Hypoxia along with reoxygenation which occurs with increased ventilation consequent to apnea, similar to ischemia-reperfusion, results in oxidative stress, inflammation and eventually endothelial dysfunction contributing to progression of $\mathrm{HF}$ as detailed elsewhere $[1,2]$. Activation of redox-sensitive genes via transcription factors such $\mathrm{NFkb}$ are involved in these processes.

As noted above, the consequences of CSA and OSA are qualitatively similar, though there are quantitative differences. One such difference is the large negative intrathoracic pressure drops during upper airway occlusion which defines obstructive apnea (Figs. 1, 2). These pressure 
changes are reflected in the surrounding of all intrathoracic structures and have significant hemodynamic consequences. Augmented gradient between intraventricular pressure and intrathoracic (juxtacardiac) pressure, which determines left ventricular transmural pressure, causes increased ventricular wall tension and oxygen consumption. Negative intrathoracic pressure increases venous return and right ventricular preload [1,2] which may cause septal deviation impairing left ventricular stroke volume. The negative pressure in the lung interstitial compartment promotes pulmonary edema (Fig. 3) [1, 2]. The described changes have unfavorable impact on left ventricular function and may cause or worsen existing HF.

Not only left ventricular, but also atrial function and structure may be affected by repetitive negative shifts in intrathoracic pressure. Augmented negative pressure gradient between intra-atrial cavites and juxtacardiac space causes atrial chamber distension and fibrosis resulting in structural atrial remodeling as well as increased $\mathrm{P}$-wave duration in ECG recognized as electrical atrial remodeling. These pathophysiological changes along with increased sympathetic activity and myocardial hypoxia are believed to be the substrate for atrial fibrillation [37].

Regarding negative pressure swings associated with CSA, these will be noted during the hyperventilation which follows the apnea; the magnitude of the negative pressure changes depend on stiffness of the lung and also the depth of inhalation.

\section{Treatment of sleep apnea in HF and its impact on cardiovascular function and mortality}

The various therapeutic approaches for both OSA and CSA have been reviewed extensively elsewhere $[1,2,38]$ and will only be briefly reviewed below.

\section{Obstructive sleep apnea}

In OSA, general recommendations include lifestyle changes such as exercise, cessation of smoking and weight loss when applicable, avoidance of alcohol, hypnotics, opioids, phosphodiesterase inhibitors, and supine position during sleep. Pharmacological approaches to treat OSA in general have not proven effective [39]. However, fluid overload should be treated aggressively. Phosphodiesterase inhibitors [40] and opioids [41] at bed time could worsen sleep apnea and should be avoided. Phosphodiesterase inhibitors perhaps via vasodilatation and vascular congestion in the nasal cavity and upper airway could promote OSA, and opioids via central mechanisms could cause central and obstructive apneas [41].

Continuous positive airway pressure (CPAP) ventilation is the treatment of choice. It provides upper airway patency (pneumatic splint) and at appropriate airway pressure keeps the upper airway open preventing development of obstructive apnea and hypopnea. In patients with OSA and $\mathrm{HF}$ with reduced ejection fraction, 3 randomized clinical trials have shown variable [42-44] but significant increases in LVEF following 1 to 3 month therapy with CPAP. In a fourth randomized study [45], ejection fraction did not change significantly; in this regard, perhaps the most important issue is adherence to CPAP [1, 2, 38, 46].

There is only one double-blind placebo-controlled (sham CPAP, a CPAP device with minimal pressure) trial in $\mathrm{HF}$ with presereved ejection fraction [47]. In this study of 12 weeks duration, effective CPAP therapy resulted in a significant increase in $\mathrm{E} / \mathrm{A}$ ratio and a significant decrease in isovolemic relaxation and mitral deceleration time [47].

In regards to mortality, observational studies [4, 5, 48, 49] suggest that HF patients with OSA have a shorter survival compared to matched patients without OSA, and that effective treatment of OSA with CPAP [5, 48, 49] improves survival, but only in those who remain adherent to CPAP. This observation that adherence to CPAP is critical for survival, is similar to that noted above for improved LVEF. The reason why many HF patients are not compliant with CPAP is that they commonly do not suffer from excessive day time sleepiness and therefore do not see any immediate improvement. This is in contrast to garden variety OSA patients whose day time sleepiness is eliminated by CPAP and therefore they remain CPAP-compliant. Adherence is the Achilles tendon of CPAP.

In the largest study from US [5] of medicare beneficiaries with newly diagnosed $\mathrm{HF}$, the survival of the 258 patients who were diagnosed with sleep apnea and were treated for it, was far superior to the other 30,065 HF patients who were not tested for sleep apnea (Fig. 4).

\section{Central sleep apnea}

Multiple studies have shown that CSA is an independent predictor of mortality in $\mathrm{HF}$ ([3], [6], [7]; for reviews see [1], [2]) (Fig. 4). In one of the studies with a large number of variables as confounders, high AHI, low diastolic blood pressure 


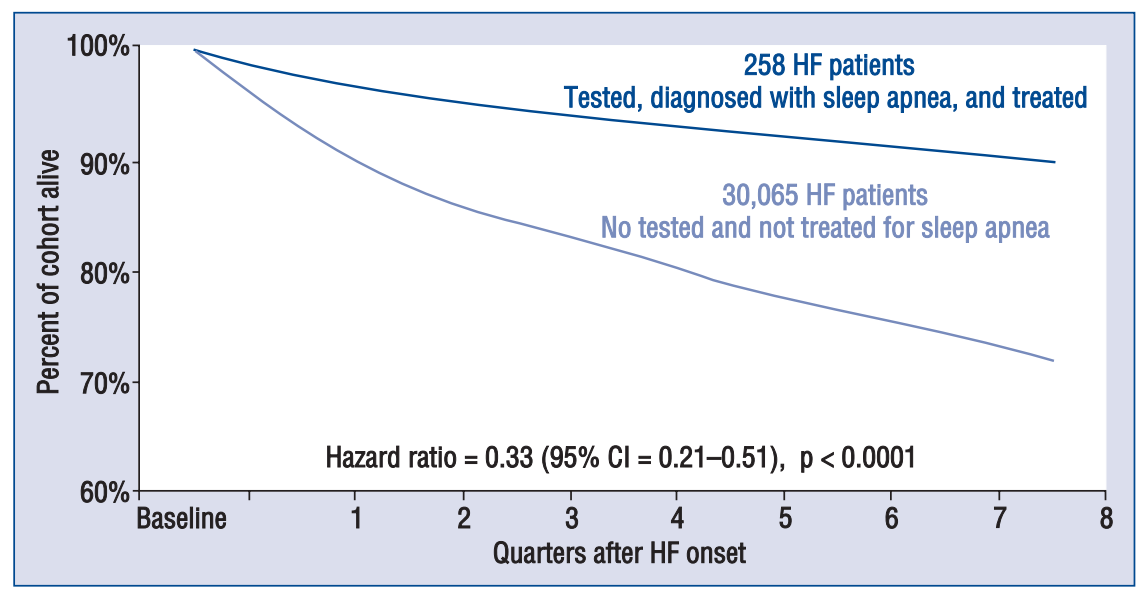

Figure 4. Kaplan-Maier survival curves, adjusted by age, gender, and Charlson Comorbidity Index, 2004-2005. Survival of heart failure (HF) patients diagnosed and treated for sleep apnea compared with the remaining patients who were not tested for sleep apnea. Modified from [5].

and right ventricular ejection fraction were the 3 variables which independently were associated with excess mortality [6].

Meanwhile, there are multiple options for treatment of CSA in HF. The severity of CSA is related to degree of left ventricular dysfunction and therefore optimization of medications and resynchronization therapy when applicable are of primary importance in management before performing a sleep study $[1,2]$. Optimal medical therapy decreases pulmonary congestion, hyperventilation, and circulation time all of which may stabilize breathing during sleep. Improvement of CSA during therapy with beta-adrenolytics has been demonstrated [50]. However, HF is a progressive disorder, and overall introduction of beta-blockers has not changed the prevalence of sleep apnea in HF (Table 1).

Meanwhile, resynchronization therapy improves CSA, which is in contrast to OSA. A recent meta-analysis [51] of 9 studies (170 patients) revealed that in patients with CSA resynchronization therapy reduced AHI significantly - mean reduction in AHI was about 13 events/h whereas in OSA it was only 3 events/h.

Currently, a few options for pharmacotherapy of CSA have been studied [52-54]. Nocturnal oxygen therapy eliminates nocturnal oxyhemoglobin desaturation, improves $\mathrm{VO}_{2} \max$ (see [2] and [52]), and also in randomized clinical trials increases LVEF and quality of life [55-57].

In small randomized clinical trials, theophylline [53] and acetazolamide [54] have been shown effective. However, their safety and long-term benefits require large trials. Theophylline stimulates breathing by competitive inhibition of adenosine from its receptors. However its pro-arrhythmogenic properties limit its use in patients with $\mathrm{HF}$. Acetazolamide induces mild metabolic acidosis and increases the difference between resting $\mathrm{PCO}_{2}$ and apnea threshold $\left(\mathrm{PCO}_{2}\right.$ reserve).

Several investigators have used CPAP to treat central apnea (see [38]). Whereas OSA is initially eliminated with overnight CPAP titration only in about $50 \%$ of patients, CPAP suppresses CSA [58]. The mechanisms include decreasing venous return and unloading the left ventricle both decreasing pulmonary capillary wedge pressure, decreasing circulation time, and increasing functional residual capacity all of which contribute to stabilization of breathing $[1,2,24]$. In addition, in some patients with CSA, upper airway closure occurs which is reversed by CPAP.

Regarding effects of CPAP on mortality, Bradley et al. [59] carried out a large prospective study with the use of CPAP in patients with HF and co-morbid CSA (The Canadian Continuous Positive Airway Pressure for Patients with Central Sleep Apnea and Heart Failure, CANPAP study). Two hundred fifty eight eligible patients were randomized to either control group with optimal medical therapy or to CPAP group with optimal medical therapy and CPAP. The average follow up time was 2 years. The study was terminated prematurely after an interim analysis, for several reasons including an early excess mortality in the CPAP arm. The reason for this excess mortality in the CPAP arm has been analyzed in detail elsewhere [60]. The major reason was that in almost $50 \%$ of the patients 
CSA was not suppressed by CPAP (these patients are considered CPAP non responders). Indeed, in a post hoc analysis, longer survival without heart transplant was reported in patients in whom CPAP effectively suppressed CSA (drop in AHI $<15 / \mathrm{h}$, mean $=6 / \mathrm{h}$ from a baseline of about $40 / \mathrm{h}$ ) in comparison to the control group [7].

For CPAP nonresponders, adaptive servoventilator (ASV) devices are the treatment of choice. The operation algorithm of these devices is quite unique and different from CPAP or bi-level devices [61]. There are 3 specific features: first, these devices stabilize the upper airway by applying a fixed (old generations) or variable expiratory positive airway pressure (automatic, like autoCPAP, new generations) to eliminate obstructive disordered breathing. Second, they act as a ventilator and deliver a mandated breath, if spontaneous breathing ceases for just few seconds. Third, they counterbalance ventilatory instability by modulating the degree of inspiratory pressure support (defined as inspiratory pressure minus expiratory pressure). In this regard, based on unique algorithms, the inspiratory positive airway pressure, and therefore the inspiratory support, varies in an anti-cyclic manner opposite to the prevailing flow or minute ventilation of the patient. Thus, tidal volume increases during hypoventilation and decreases during hyperventilation. This component of ventilation that is anti-cyclic to the periodicity of the patient's own breathing, acts to dampen the oscillations in the ventilatory drive that underlie periodic breathing.

Teschler et al. [62] are credited with the first ASV study showing that in a 5 arm overnight study, ASV was more effective than oxygen, CPAP and bi-level therapy. In a meta-analysis of multiple studies using ASV as a contrast group $\left(\mathrm{O}_{2}\right.$, CPAP, or continued medical therapy), will show that ASV improves AHI and LVEF significantly [63]. Two observational long-term studies $[8,10]$ have demonstrated improved survival in those who used the ASV device compared to those who refused. Currently, 2 large prospective trials are ongoing - SERVE-HF and ADVENT-HF. Patients with $\mathrm{HF}$ and coexisting sleep apnea (in the first study CSA, in the second CSA or OSA) are randomized into 2 groups: the first group on optimal medical therapy for HF or the second group treated additionally with ASV. The results of these studies should answer the question whether ventilation by means of ASV influences long term prognosis and frequency of hospital re-admissions.
Despite proven beneficial effects of CPAP and also ASV therapy, a number of patients cannot tolerate mask/positive pressure device therapy; as noted before, most HF patients with sleep apnea do not see immediate improvement with the use of these devices. We believe this is the major factor influencing non-adherence. Therefore new therapeutic methods are currently a subject of research. An important advancement is transvenous phrenic nerve stimulation, which is easily placed by an electrophysiologist. This acts like a pacemaker stimulating the phrenic nerve during sleep. In a preliminary study [64] a temporary transvenous lead was placed in either the right brachiocephalic or left pericardiophrenic vein in 13 patients with $\mathrm{HF}$ and confirmed CSA. During PSG on therapy night, when phrenic nerve stimulation was applied, a significant decrease in AHI and central apnea index was observed when compared to control night without phrenic nerve stimulation. Simultaneously, arousal index related to periodic breathing significantly decreased. This novel method is important for a variety of reasons including the ease of use by the patient which should improve adherence, an important drawback of mask/positive airway therapy. Long-term studies with phrenic nerve stimulation are pending. Finally we note that cardiac transplantation virtually eliminates CSA indicating that CSA is the consequence of $\mathrm{HF}$ [65]. Unfortunately, however, a large number of cardiac-transplant recipients gain weight and develop OSA.

\section{Conclusions}

Despite recent advances in HF management, quality of life, hospital readmissions and mortality remain unacceptably high. Therefore, an increasing focus on treating co-morbidities which could change such grim prognosis is clinically relevant. SDB is reported to be among most common co-morbidities affecting $\mathrm{HF}$ patients. It occurs in at least half of $\mathrm{HF}$ population and is associated with more severe HF symptoms, lower ejection fraction and higher prevalence of arrhythmia. SDB has also been proven to be an independent risk factor of poor outcome. There are documented treatment options based on positive airway pressure ventilation, which successfully reduce number of apnea episodes, but also improve left ventricular systolic function, exercise tolerance and possibly long term prognosis. Due to low recognition of SBD it seems necessary to introduce routine investigations in patients with $\mathrm{HF}$, especially with new promising treatment methods such as phrenic nerve stimulation. 
Conflict of interest: Prof. P. Ponikowski is a member of speaker's bureau and a consultant for Respicardia. Prof. S. Javaheri is a consultant for Respicardia, receives honoraria for lectures from Res-Med, Philips-Respironics, Respicardia, and received a grant from Philips-Respironics. Dr R. Germany is Chief Medical Officer at Respicardia. Respicardia supported the research study on prevalence of sleep disordered breathing in patients with heart failure conducted by I. Flinta, M. Krawczyk, P. Ponikowski.

\section{References}

1. Javaheri S. Sleep-related breathing disorders in heart failure. In: Douglas L. Mann ed. Heart failure. A companion to Braunwald's heart disease. WB Saunders, Philadelphia 2010; 471-487.

2. Javaheri S. Heart failure. In: Kryger MH, Roth T, Dement WC eds. Principles and practices of sleep medicine. WB Saunders, Philadelphia 2011: 1400-1415.

3. Lanfranchi PA, Braghiroli A, Bosimini E et al. Prognostic value of nocturnal Cheyne-Stokes respiration in chronic heart failure. Circulation, 1999; 99: 1435-1440.

4. Wang H, Parker JD, Newton GE et al. Influence of obstructive sleep apnea on mortality in patients with heart failure. J Am Coll Cardiol, 2007;49:1625-31.

5. Javaheri S, Caref EB, Chen E, Tong KB, Abraham WT. Sleep apnea testing and outcomes in a large cohort of medicare beneficiaries with newly diagnosed heart failure. Am J Respir Crit Care Med, 2011; 183: 539-546.

6. Javaheri S, Shukla R, Zeigler H, Wexler L. Central sleep apnea, right ventricular dysfunction and low diastolic blood pressure are predictors of mortality in systolic heart failure. J Am Coll Cardiol, 2007; 49: 2028-2038.

7. Arzt M, Floras JS, Logan AG et al. Suppression of central sleep apnea by continuous positive airway pressure and transplant-free survival in heart failure: A post hoc analysis of the Canadian Continuous Positive Airway Pressure for Patients with Central Sleep Apnea and Heart Failure Trial (CANPAP). Circulation, 2007; 115: 3173-3180.

8. Takama N, Kurabayashi M. Effect of adaptive servo-ventilation on 1 year prognosis in heart failure patients. Circ J, 2012; 76: 661-667.

9. Hanly P, Zuberi-Khkhar N. Increased mortality associated with Cheyne-Stokes respiration in patients with congestive heart failure. Am J Respir Crit Care Med, 1996; 153: 272.

10. Jilek C, Krenn M, Sebah D et al. Prognostic impact of sleep disordered breathing and its treatment in heart failure: an observational study. Eur J Heart Fail 2011;13(1):68-75.

11. The Report of an American Academy of Sleep Medicine Task Force. Sleep-related breathing disorders in adults: recommendations for syndrome definition and measurement techniques in clinical research. Sleep, 1999; 22: 667-689.

12. Javaheri S, Parker TJ, Wexler L et al. Occult sleep-disordered breathing in stable congestive heart failure. Ann Intern Med, 1995; 122: 487-492 (Erratum, Ann Intern Med 1995; 123: 77).

13. Javaheri S. Sleep disorders in systolic heart failure: A prospective study of 100 male patients. The Final Report. Int J Cardiol, 2006; 106:21-28.

14. Ng AC, Freedman SB. Sleep disordered breathing in chronic heart failure. Heart Fail Rev, 2009; 14: 89-99.
15. Scharf C, Cho YK, Bloch KE et al. Diagnosis of sleep-related breathing disorders by visual analysis of transthoracic impedance signals in pacemakers. Circulation, 2004; 110: 2562-2567.

16. S Vazir A, Hastings PC, Dayer M et al. A high prevalence of sleep disorder breathing in men with mild symptomatic chronic heart failure due to left ventricular systolic dysfunction. Eur J Heart Failure, 2007; 9: 243-250.

17. Oldenburg O, Lamp B, Faber L, Teschler H, Horstkotte D, Topfer V. Sleep-disordered breathing in patients with symptomatic heart failure: A contemporary study of prevalence in and characteristics of 700 patients. Eur J Heart Fail, 2007; 9: 251-257.

18. MacDonald M, Fang J, Pittman SD, White DP, Malhotra A. The current prevalence of sleep disordered breathing in congestive heart failure patients treated with beta-blockers. J Clin Sleep Med, 2008; 4: 38-42.

19. Ferreira S, Marinho A, Patacho $M$ et al. Prevalence and characteristics of sleep apnoea in patients with stable heartfailure: Results from a heart failure clinic. Pulmonary Med, 2010; 10: 1-9.

20. Hagenah G, Beil D. Prevalence of Cheyne-Stokes respiration in modern treated congestive heart failure. Sleep Breath, 2009; 13: 181-185.

21. Bitter T, Faber L, Hering D, Langer C, Horstkotte D, Oldenburg O. Sleep-disordered breathing in heart failure with normal left ventricular ejection fraction. Eur J Heart Fail, 2009; 11: 602-608.

22. Ward M. Periodic respiration. A short historical note. Ann R Coll Surg Engl, 1973; 52: 330-334.

23. Cheyne J. A case of apoplexy in which the fleshy part of the heart was converted into fat. Dublin Hospital Reports, 1818; 2: 216-223.

24. Javaheri S, Dempsey JA. Central sleep apnea. Comprehensive Physiology, 2013; 3: 141-163.

25. Xie A, Skatrud JB, Puleo DS, Rahko PS, Dempsey JA. Apnea-hypopnea threshold for $\mathrm{CO} 2$ in patients with congestive heart failure. Am J Respir Crit Care Med, 2002; 165: 1245-1250.

26. Javaheri S. A mechanism of central sleep apnea in patients with heart failure. N Eng J Med, 1999; 341: 949-954.

27. Ponikowski P, Chua TP, Anker SD et al. Peripheral chemoreceptor hypersensitivity: an ominous sign in patients with chronic heart failure. Circulation, 2001; 104: 544-549.

28. Yumino D, Redolfi S, Ruttanaumpawan P et al. Nocturnal rostral fluid shift: A unifying concept for the pathogenesis of obstructive and central sleep apnea in men with heart failure. Circulation, 2010; 121: 1598-1605.

29. Javaheri S, Corbett WS. Association of low arterial $\mathrm{PCO}_{2}$ with central sleep apnea and ventricular arrhythmias in ambulatory patients with stable heart failure. Ann Intern Med, 1998; 128: 204-207.

30. Javaheri S, Almoosa KF, Saleh K, Mendenhall CL. Hypocapnia is not a predictor of central sleep apnea in patients with cirrhosis. Am J Respir Crit Care Med, 2005; 171: 908-911.

31. Xie A, Skatrud JB, Barczi SR et al. Influence of cerebral blood flow on breathing stability. J Appl Physiol, 2009; 106: 850-856.

32. Georgiadis D SM, Werdan K. Cerebrovascular reactivity is impaired in patients with cardiac failure. Eur Heart J, 2000; 21: 407-413.

33. Jordan AS, White DP. Pharyngeal motor control and the pathogenesis of obstructive sleep apnea. Respir Physiol Neurobiol, 2008; 160: 1-7.

34. Javaheri S, Parker TJ, Liming JD et al. Sleep apnea in 81 ambulatory male patients with stable heart failure. Types and their prevalences, consequences, and presentations. Circulation, 1998; 97: 2154-2159. 
35. Ponikowski P, Anker SD, Chua TP et al. Oscillatory breathing patterns during wakefulness in patients with chronic heart failure: clinical implications and role of augmented peripheral chemosensitivity. Circulation, 1999; 100: 2418-2424.

36. Javaheri S. Shukla R, Wexler L. Association of smoking, sleep apnea and plasma alkalosis with nocturnal ventricular arrhythmias in men with systolic heart failure. Chest, 2012; 141: 1449-1456.

37. Baranchuk A. Sleep apnea, cardiac arrhythmias, and conduction disorders. J Electrocardiol, 2012; 45: 508-512.

38. Javaheri S. Treatment of obstructive and central sleep apnea in heart failure: Practical options. Eur Respir Rev, 2007; 16: 183-188.

39. Kohler M, Stradling JR. Pitfalls of clinical trials on pharmacological treatment for obstructive sleep apnoea: future directions. Expert Opin Investig Drugs, 2011; 20: 1033-1037.

40. Roizenblatt S, Guilleminault C, Poyares D, Cintra F, Kauati A, Tufik S. A double-blind, placebo-controlled, crossover study of sildenafil in obstructive sleep apnea. Arch Intern Med, 2006; 166: 1763-1767.

41. Cao M, Javaheri S. Chronic opioid use: Effects on respiration and sleep. In: Tvildiani D, Gegechkori K eds. Opioids pharmacology, clinical uses and adverse effects. Edited by Nova Science Publishers, Inc., NewYork 2012: 1-13.

42. Kaneko Y, Floras JS, Usui K et al. Cardiovascular effects of continuous positive airway pressure in patients with heart failure and obstructive sleep apnea. N Engl J Med, 2003; 248: 1233-1241.

43. Mansfield DR, Gollogly C, Kaye DM. Controlled trail of continuous positive airway pressure in obstructive sleep apnea and heart failure. Am J Respir Crit Care Med, 2004; 169: 361-366.

44. Egea CJ, Aizpuru F, Pinto JA et al. Cardiac function after CPAP therapy in patients with chronic heart failure and sleep apnea: A multicenter study. Sleep Med, 2008; 9: 660-666.

45. Smith LA, Vennelle M, Gardner RS et al. Auto-titrating continuous positive airway pressure therapy in patients with chronic heart failure and obstructive sleep apnoea: A randomized placebo-controlled trial. Eur Heart J, 2007; 28: 1221-1227.

46. Javaheri S. Heart failure. In: Kushida CA ed. The encyclopedia of sleep. Academic Press, Waltham, MA 2013; 3: 374-386.

47. Arias MA, Garcia-Rio F, Alonso-Fernandez A et al. Obstructive sleep apnea syndrome affects left ventricular diastolic function. Circulation. 2005;112:375-383.

48. Kasai T, Narui K, Dohi P et al. Prognosis of patients with heart failure and obstructive sleep apnea treated with continuous positive airway pressure. Chest, 2008; 133: 690-696.

49. Damy T, Margarit L, Noroc A, Bodez D et al. Prognostic impact of sleep-disordered breathing and its treatment with nocturnal ventilation for chronic heart failure. Eur J Heart Fail, 2012; 14: 1009-1019.

50. Tamura A, Kawano Y, Naono S, Kotoku M, Kadota J. Relationship between beta-blocker treatment and the severity of central sleep apnea in chronic heart failure. Chest, 2007; 131: 130-135.
51. Lamba J, Simpson CS, Redfearn DP, Michael KA, Fitzpatrick M, Baranchuk A. Cardiac resynchronization therapy for the treatment of sleep apnoea: a meta-analysis. Europace 2011;13: $1174-9$.

53. Javaheri S, Parker TJ, Wexler L, Liming JD, Lindower P, Roselle GA. Effects of theophylline on sleep disordered breathing in stable heart failure: A prospective, double-blind, placebo controlled, crossover study. N Engl J Med, 1996; 335: 562-567.

54. Javaheri S. Acetazolamide improves central sleep apnea in heart failure: A double-blind prospective study. Am J Respir Crit Care Med, 2006; 173: 234-237.

52. Javaheri S. Pembrey's dream: The time has come for a long-term trial of nocturnal supplemental nasal oxygen to treat central sleep apnea in congestive heart failure. Chest, 2003; 123: 322-325.

55. Sasayama S, Izumi T, Seino Y et al. Effects of nocturnal oxygen therapy on outcome measures in patients with chronic heart failure and Cheyne-Stokes respiration. Circ J, 2006; 70: 1-7.

56. Sasayama S, Izumi T, Matsuzaki M et al. Improvement of quality of life with nocturnal oxygen therapy in heart failure patients with central sleep apnea. Circ J, 2009; 73: 1255-1262.

57. Toyama T, Seki R, Kasama $\mathrm{S}$ et al. Effectiveness of nocturnal home oxygen therapy to improve exercise capacity, cardiac function and cardiac sympathetic nerve activity in patients with chronic heart failure and central sleep apnea. Circ J, 2009; 73: 299-304.

58. Javaheri S. Effects of continuous positive airway pressure on sleep apnea and ventricular irritability in patients with heart failure. Circulation, 2000; 101: 392-397.

59. Bradley TD, Logan AG, Kimoff RJ et al. Continuous positive airway pressure for central sleep apnea and heart failure. $\mathrm{N} \mathrm{Eng}$ J Med, 2005; 353: 2025-2033.

60. Javaheri S. CPAP should not be used for central sleep apnea in congestive heart failure patients. J Clin Sleep Med, 2006; 15: 399-402.

61. HarrisN.JavaheriS.AdvancedPAPtherapies.In:MatticeC,BrooksR, Lee-Chiong T eds. Fundamentals of sleep technology. Lippincott, Williams and Wilkins, Philadelphia 2012; 444-452.

62. Teschler H, Döhring J, Wang YM et al. Adaptive pressure support servo-ventilation. Am J Respir Crit Care Med, 2001; 164: 614 .

63. Sharma BK, Bakker JP, McSharry DG, Desai AS, Javaheri S, Malhotra A. Adaptive servo-ventilation for treatment of sleepdisordered breathing in heart failure: A systematic review and meta-analysis. Chest, 2012; 142: 1211-1221.

64. Ponikowski P, Javaheri S, Michalkiewicz D et al. Transvenous phrenic nerve stimulation for the treatment of central sleep apnoea in heart failure. Eur Heart J, 2012; 33: 889-894.

65. Javaheri S, Abraham W, Brown C et al. Prevalence of obstructive sleep apnea and periodic limb movement in 45 subjects with heart transplantation. Eur Heart J, 2004; 25: 260-266. 